\title{
Special section on the role of information technology in service delivery
}

\author{
Jaeki Song $^{1}$ • Jeff Baker ${ }^{2}$ - Fred D. Davis ${ }^{3}$ \\ Published online: 8 April 2015 \\ (C) Springer Science+Business Media New York 2015
}

\section{Introduction}

Much of the radical transformation of the world economy from an industrial, manufacturing-oriented economy to a post-industrial, service-oriented one comes from the application of information technology (IT) to service delivery (Rai and Sambamurthy 2006). IT has contributed - and continues to contribute - to increased efficiency and effectiveness in service delivery. IT also reduces the cost of service and creates additional service-related revenues for firms. Furthermore, IT enables the development of new services. Each of these effects that result from the use of IT in service delivery have the potential to add value for customers and create competitive advantage for firms.

Service, and the development and delivery of services, has been studied in several fields, including marketing, operations management, economics, and information systems (Rai and

Jaeki Song

jaeki.song@ttu.edu

Jeff Baker

jbaker@aus.edu

Fred D. Davis

fdavis@walton.uark.edu

1 Rawls College of Business Administration, Texas Tech University, Lubbock, TX 79409-2101, USA

2 School of Business and Management, American University of Sharjah, PO Box 26666, Sharjah, UAE

3 Department of Information Systems, Sam M. Walton College of Business, Business Building 301, Fayetteville, AR 72701, USA

Sambamurthy 2006). Researchers have provided extensive reviews of service, defining it as a capability or capabilities that one economic entity provides to other entities as part of the value-creation process (Vargo and Lusch 2004). This process of service creation and delivery can be simple and routine, or can be complex and innovative. Regardless of the characteristics of the service being provided, the needs of many service providers can be addressed by advanced IT (Jorgenson 2001). The contribution of IT to service delivery is particularly apparent in web services, computing services, business intelligence services, and information technology infrastructure. And for the consumer, IT-enabled service delivery can be seen in fields as diverse as healthcare, travel, retailing, media, and entertainment. Service-oriented IT is thus helping to determine the directions in which numerous industries - and the world economy in general - will grow and develop in the future. The depth and the diffusion of technology is helping to accelerate the application and use of knowledge in ways unimagined only a decade ago.

The main purpose of this special section is to examine the role of IT in delivering service. It provides a venue in which new opportunities, challenges, and solutions are introduced to researchers. This special section has taken a broad view of service, centering on how service entities utilize IT to create value for individuals, firms, collectives, and/or ecosystems. Foregoing research has considered contexts for service delivery such as healthcare, financial services, education, social services, and government programs at various levels. Several of these contexts appear here. Speaking generally, this special section focuses on the role of IT in the delivery of services, cost-effective service delivery, IT enabled service design and development, facilitating service delivery through IT processes and practices, and the investigation and implications of value co-creation for IT-based service delivery in service innovations. 


\section{Research issues}

Numerous issues related to IT in service delivery are being explored by researchers. One foundational issue is the locus of service creation. The vast majority of extant research is built upon a closed model that emphasizes interaction between products and services inside of an organization. This model has emerged largely from a goods-dominant logic, and initially focused on how services add value to goods. A logical extension of the closed model would be to examine factors outside the organization, and to do so from a service-dominant logic perspective. In service-dominant logic, a service itself can generate value and goods may support the service. Furthermore, an open model can examine participants in the service-creation process that are outside the organization, and can examine the network effects that are germane to services.

This open model presents researchers with additional issues on which to focus, including the issues of intangibility, heterogeneity, inseparability, and perishability. When considering goods only, productivity can be calculated in a straightforward manner as output/input. For example, if line worker produces $10 \mathrm{TVs}$ per day, his or her productivity is 10 . An underlying assumption is that the quality of the product should be maintained constant. When considering services, or when considering good-service bundles, however, the assumption of constant quality becomes questionable because of the intangibility, heterogeneity, inseparability, and perishability of services. Maximizing the productivity of new and redesigned services is in part dependent on identifying ways to measure productivity.

Another research issue is the role and function of service providers. Services are the outcome of the interactions between providers and recipients. This has been reflected in the stream of research that examines the way to increase the efficiency of service processes by co-creating value with customers. One of the challenges in this area is to figure out how customers define and obtain value by participating in the service processes. Answers to this problem will help develop an understanding of customers' perceptions about service quality so that firms can modify their service strategies accordingly. It will also be valuable to investigate how service providers initiate the value-creation process, but still involve customers.

Still another research issue is the development and growth of IT-based services for Web technologies. Many studies have investigated the effectiveness of IT when delivering services on the Web (e.g., Song et al. 2007, Bharati and Chaudhury 2004). The use of cloud computing has been one particular focus, with researchers observing an improvement in service as well as a corresponding increase in the responsibility of the vendor (Vouk 2008, Chen et al. 2012). With the development of mobile and cloud computing, location based services, and near field communication services, new opportunities exist to provide information to customers. Some have noted that the growth of cloud computing changes businesses' scope by creating value throughout the services development and delivery process, which in turn significantly impacts firm performance (Bharadwaj et al. 2013). Similarly, researchers have noted that services can now be provided at notably lower cost (Marston et al. 2011).

Other researchers have noted that the advent of cloud computing represents a fundamental change in IT, with new strategic options available to firms. Cloud computing not only enables stand-alone services based on IT, but also enables the growth of entire business ecosystems, such as the well-known Apple hardware, software, media, and mobile application ecosystem. Firms need to consider the factors in their competitive environment and the technical elements of infrastructure as they choose which existing ecosystems to participate in - or choose to build their service portfolio into their own full-fledged business ecosystems. If investment in platform-based IT services business is made under the ecosystems rather than an individual investment, economies of scale can be achieved. These strategic considerations require economic analysis as well as technical feasibility analysis.

These as well as other issues will continue to be a part of the research agenda for service delivery through IT.

\section{Development of the special section}

For the special section, a call for papers was posted in early 2013 and submissions were sought from the community of IS scholars. In addition to the open solicitation of submissions, key papers that were relevant to the special section theme that were presented at the 2013 Pacific-Asia Conference on Information Systems (PACIS) were invited. All of the papers went through two rounds of review; with two having an additional round of review to address final issues. Regular submissions as well as invited submissions appear in the special issue. Multiple methodological approaches are featured in the special section. The papers include a field study (Seethamraju 2015), a design-science paper (Nguyen et al. 2015), an empirical study (Tang et al. 2015), and an essay (Kim et al. 2015). Brief descriptions of the papers now follow, with the papers themselves appearing later in this issue. 


\section{Papers in the special section}

Ravi Seethamraju ("Adoption of Software as a Service Enterprise Resource Planning Systems in Small and Medium Sized Enterprises") investigates the factors that influence the adoption of enterprise resource planning (ERP) systems. More specifically, he investigates ERP systems that are offered in the cloud under the software as a service (SaaS) model. He highlights the advantages of these types of systems as well as the challenges associated with their adoption in a cross-sectional field study of four organizations. Key factors influencing adoption include the reputation of the software vendor, the fit of the software to the business, the potential willingness of the vendor to support the customer throughout the life cycle of the product, the co-creation of value between vendors and customers, and the general benefits of integrated ERP systems. The willingness of the software vendor to work with the firm to address issues and co-create value during the implementation and post-implementation phases are also indicated to be beneficial. The firms' competitive pressures, concerns about data security, and system performance were relatively less important in this study. In fact, many firms found the cloud-based ERP solutions to be safer and more reliable than their current systems, reducing the potential downside to adoption.

Bang Nguyen, Frada Burstein, and Julie Fisher ("Improving Service of Online Health Information Provision: A Case of Usage-Driven Design for Health Information Portals") describe ways to improve health information portals to provide a better search experience for users. Taking a design-science approach, they analyze usage data to address problems encountered during searches for information. They then develop a conceptual approach and an architecture to support system capabilities. Specifically, they propose a content issue reporting application and a topic search application. In addition to the practical and technical contribution of their work, the conceptual contribution of this paper is a demonstration and explanation of how usage data can be analyzed to enhance system functionality and improve service delivery.

Jian Tang, Ping Zhang, and Philip Fei Wu ("Categorizing Consumer Behavioral Responses and Artifact Design Features: The Case of Online Advertising") consider the design features of advertising and the various types of responses that consumers have to it. They categorize the features of online advertisement in terms of ad content, which they define as the message or meaning an ad carries; ad form, which is the presentation style; and ad action, which includes the behaviors of an online ad such as movement and frequency of appearance. The authors also explain that when encountering ads, consumers respond with either approach or avoidance. These two actions of approach or avoidance can be either active or passive. Thus, consumers can have an active approach, a passive approach, an active avoidance, or a passive avoidance response to an ad. These authors present empirical evidence to support their categorizations for the features of online ads as well as for the four responses to online ads. Their descriptive work sets the stage for other researchers to examine these categories and responses in the future.

Miri Kim, Jaeki Song, and Jason Triche ("Toward an Integrated Framework for Innovation in Service: A ResourceBased View and Dynamic Capabilities Approach") present an explanation of how firms' internal resources and relational capabilities interact and evolve to enable service innovation. The authors build on the resource-based view of the firm (RBV), the dynamic capabilities framework (DCF), and service innovation literature to develop their research model and propositions.

The authors classify organizational resources as being either property-based resources or knowledge-based resources. Property-based resources may be either 'discrete' or 'systemic', while knowledge-based resources are either 'functional' or 'organizational'. These resources, in combination with relational capabilities, serve as inputs that enable the three dynamic service capabilities of integration, reconfiguration, and extraction. These dynamic service capabilities then support the process of service innovation. This essay provides suggestions for how researchers may choose to investigate its propositions and also provides practical guidance for managers to assess their resources and capabilities to develop new service offerings.

\section{Conclusion}

The service-oriented economy represents a new paradigm within the economy as a whole. Service-related IT innovations are a part of the global transition from simply an information society to one that is truly knowledge-based. IT has the potential to create a virtuous cycle of learning and growth toward knowledge-based services that can add value to the economy.

As this new paradigm continues to develop, the role of IT within the economy seems likely to grow. IT development for service delivery is occurring in a number of industries - from appliances and automobiles, to watches and glasses and wearable IT, to media production and delivery, to travel and tourism, to urban infrastructure and government services. These IT developments are enabling changes in usage, safety, productivity, communication, and innovation. IT-enabled services can be delivered through computers, mobile devices, or by customer service personnel. Regardless of the industry and manner of delivery, the role of IT in services is growing. IT seems to clearly be playing a role in the shift from productcentric to service-centric business models, and in the creation of new opportunities for businesses. 


\section{References}

Bharadwaj, A., El Sawy, O. A., Pavlou, P. A., \& Venkatraman, N. (2013). Digital business strategy: toward a next generation of insights. MIS Quarterly, 37(2), 471-482.

Bharati, P., \& Chaudhury, A. (2004). An empirical investigation of decision-making satisfaction in web-based decision support systems. Decision Support Systems, 37(2), 187-197.

Chen, H., Chiang, R. H. L., \& Storey, V. C. (2012). Business intelligence and analytics: from big data to big impact. MIS Quarterly, 36(4), $1165-1188$.

Jorgenson, D. W. (2001). Information technology and the U.S. economy. American Economic Review, 91(1), 1-32.

Kim, M., Song, J., \& Triche, J. (2015). Toward an integrated framework for innovation in service: a resource-based view and dynamic capabilities approach. Information Systems Frontiers, 17(3). doi: 10. 1007/s10796-014-9505-6.

Marston, S., Li, Z., Bandyopadhyay, S., Zhang, J., \& Ghalsasi, A. (2011). Cloud computing: the business perspective. Decision Support Systems, 51(1), 176-189.

Nguyen, B.V., Burstein, F., \& Fisher, J. (2015). Improving service of online health information provision: a case of usage-driven design for health information portals. Information Systems Frontiers, 17(3). doi: 10.1007/s10796-014-9507-4.

Rai, A., \& Sambamurthy, V. (2006). Editorial notes - The growth of interest in services management: opportunities for information systems scholars. Information Systems Research, 17(4), 327331.

Seethamraju, R. (2015). Adoption of software as a service (saas) enterprise resource planning (ERP) systems in small and medium sized enterprises. Information Systems Frontiers, 17(3). doi: 10.1007/ s10796-014-9506-5.

Song, J., Jones, D., \& Gudigantala, N. (2007). The effect of incorporating compensatory choice strategies in web-based consumer decision support systems. Decision Support Systems, 43(2), 359-374.

Tang, J., Zhang, P., \& Wu, P.F. (2015). Categorizing consumer behavioral responses and artifact design features: the case of online advertising
Information Systems Frontiers, 17(3). doi: 10.1007/s10796-0149508-3.

Vargo, S. L., \& Lusch, R. F. (2004). Evolving to a new dominant logic for marketing. Journal of Marketing, 68(1), 1-17.

Vouk, M. A. (2008). Cloud computing - issues, research, and implications. Journal of Computing and Information Technology, 16(4), 235-246.

Jaeki Song is an associate professor and the Jerry S. Rawls Endowed Professor of MIS at the Rawls College of Business at Texas Tech University. His primary area of research is the innovations in Information Systems. Specific research issues include service innovations, diffusion of information technologies, social and economic consequences of information technologies, and business analytics. His research findings have appeared more than 40 refereed journal papers in Management Science, Journal of Management Information Systems, Journal of the Association for Information Systems, IEEE Transactions on Professional Communication, Decision Support Systems, Information \& Management, Information Systems Frontier, and other decent journals.

Jeff Baker is an Associate Professor at the American University of Sharjah in the United Arab Emirates. He is also a Research Associate at the Institute for Internet Buyer Behavior at Texas Tech University. His research focuses on IT strategy and electronic commerce. His research has appeared in the Journal of the AIS, Communications of the AIS, Electronic Commerce Research, the Journal of Electronic Commerce in Organizations, the International Journal of E-Business Research, and Recent Patents on Computer Science.

Fred Davis is Distinguished Professor and David D. Glass Chair in Information Systems at the Walton College of Business, University of Arkansas. He received his Ph.D. from MIT, and he has served on the business school faculties of the Universities of Michigan, Minnesota, and Maryland. His research interests include user acceptance of information technology, technology supported decision making, computer training and skill acquisition, and NeuroIS. 